\title{
Brain Cortical Structure and Executive Function in Children May Be Influenced by Parental Choices of Infant Diets
}

\author{
(D)T. Li, (D)T.M. Badger, (D)B.J. Bellando, (D) S.T. Sorensen, (D)X. Lou, and (D) X. Ou
}

\begin{abstract}
BACKGROUND AND PURPOSE: While it is known that breastfeeding promotes healthy brain development in children, the potential effects of formulas substantially differing in composition (ie, milk-based versus soy-based) during infancy on brain development are unclear.

MATERIALS AND METHODS: Seventy-one 8-year-old children who were predominantly breastfed, milk formula fed, or soy formula fed during infancy were recruited for an MR imaging examination of the brain and a Behavior Rating Inventory of Executive Function assessment (completed via a questionnaire to the parents). Brain cortical features measured from MR imaging such as cortical thickness and surface area were extracted and compared among groups and correlated with Behavior Rating Inventory of Executive Function test scores.

RESULTS: Clusters in the frontal and occipital lobes showed significant differences (cluster-wise $P \leq .05$, corrected for multiple comparisons) in cortical thickness or surface area among the 3 diet groups. The effects were more prominent for boys, particularly for comparison of the milk formula fed versus soy formula fed boys. Assessments of executive function and behavior showed significantly lower Behavior Rating Inventory of Executive Function test scores in soy formula fed versus milk formula fed groups, which were mostly attributed to differences in boys. There were no differences between milk formula fed and breastfed groups for either sex. Mean cortical thickness for several of the clusters in the brain showing infant diet-associated effects significantly correlated with Behavior Rating Inventory of Executive Function scores.
\end{abstract}

CONCLUSIONS: Choices of infant diets (ie, breastfed, milk formula fed, soy formula fed) may have long-term and sex-specific effects on the cortical development and executive function and behavior of children's brains.

ABBREVIATIONS: $\mathrm{BF}=$ breastfed; BRIEF = Behavior Rating Inventory of Executive Function; $\mathrm{MF}=$ milk formula fed; $\mathrm{SF}=$ soy formula fed

$\mathbf{T}$ he World Health Organization recommends exclusive breastfeeding for neonates to up to 6 months of age and continued breastfeeding in combination with other foods to age 2 years or

Received December 27, 2019; accepted after revision April 17, 2020.

From the Departments of Radiology (T.L., X.O.) and Pediatrics (T.M.B., B.J.B., S.T.S., X.O.), University of Arkansas for Medical Sciences, Little Rock, Arkansas; Arkansas Children's Nutrition Center (T.L., T.M.B., B.J.B., S.T.S., X.O.), Little Rock, Arkansas; Department of Information Sciences (T.L., X.O.), University of Arkansas at Little Rock, Little Rock, Arkansas; Department of Biostatistics (X.L., X.O.), University of Florida, Gainesville, Florida; and Arkansas Children's Research Institute (X.O.), Little Rock, Arkansas.

This project was supported by US Department of Agriculture-Agricultural Research Service Project 6026-51000-010-05S at the Arkansas Children's Nutrition Center. The Principal Investigator is also supported by National Institutes of Health R01 HD099099.

Please address correspondence to Xiawei Ou, PhD, 1 Children's Way, Slot 105, Little Rock, AR 72202; e-mail: ouxiawei@uams.edu

-- Indicates open access to non-subscribers at www.ajnr.org

Indicates article with supplemental on-line table.

Indicates article with supplemental on-line photos.

http://dx.doi.org/10.3174/ajnr.A6601 older. The American Association of Pediatrics also reaffirmed its breastfeeding guidelines, which are essentially consistent with the World Health Organization recommendations. ${ }^{1}$ With breastfeeding support from all sectors of society, the breastfeeding rate in the United States has been increasing in recent years. The most recent Centers for Disease Control and Prevention Breastfeeding Report Card shows that currently, $83.2 \%$ of children born in United States started out breastfeeding, $46.9 \%$ of all infants were exclusively breastfed at 3 months, and $57.6 \%$ of all infants were at least partially breastfed at 6 months. ${ }^{2}$ For those who did not start out breastfeeding or ended sooner than recommended, cow's milk protein-based formula has been the main alternative. Soy proteinbased formula currently accounts for a small percentage of the infant formula market in the United States but remains useful for infants allergic to milk formula or for parents wishing to maintain a vegetarian lifestyle. Soy-based formula and milk-based formula sold in the United States meet specific requirements of the US Food and Drug Administration for nutrient content. A major difference in soy formula and all other formulas is that the soy protein 
used in soy-based formula contains isoflavones, which can have weak estrogenic activity, and this has led some health professionals to be concerned about sexual development and reproduction in children fed soy formula. Nevertheless, there is no conclusive evidence from the current literature to show adverse effects on human development, reproduction, or endocrine function of dietary soy isoflavones. ${ }^{3}$

Many studies have observed benefits to children's health and development associated with breastfeeding, including promotion of neurodevelopment such as higher intelligence. ${ }^{4,5}$ Recent neuroimaging studies have also revealed the effects of breastfeeding or breast milk intake on children's brain development, including increased total brain volume and white matter volume associated with a higher percentage of breast milk intake in boys born preterm; ${ }^{6}$ a positive association between the duration of exclusive breastfeeding and cortical thickness in the superior and inferior parietal lobes; ${ }^{7}$ better white matter development in frontal and association brain regions associated with exclusive breastfeeding; ${ }^{8}$ as well as higher regional gray matter volume in the inferior temporal lobe and superior parietal lobe and higher white matter integrity in the left brain hemisphere (in boys) in breastfed-versus-formula fed children. ${ }^{9,10}$ Few studies have evaluated whether there are brain developmental differences associated with soy- versus milk-based formulas, despite findings that estrogen may play an important role in neurodevelopment ${ }^{11}$ and soy isoflavone may have important implications in cognitive function. ${ }^{12}$ To our knowledge, the only published data on soy formula-brain effects were electroencephalographic and event-related potential evaluations of cortical activity, in which differences in brain electrical activity associated with infant diets were observed during the first year of life. ${ }^{13,14}$

Infant diets may have long-lasting effects on brain anatomy and function and neurobehavioral outcomes, through programming mechanisms that remain to be fully elaborated. In this study, we hypothesized that there are structural differences in the brain cortex of school-age children that are associated with type of diet during infancy, ie, breastmilk, cow's milk formula, or soy formula. We also hypothesized that these brain structure differences are associated with differences in behavior, particularly, parentreported executive functioning. To test the hypothesis, we recruited healthy 8-year-old children who were predominately breastfed (BF), cow's milk formula fed (MF), or soy formula fed (SF) during the first year of life and evaluated their brain cortical development using MR imaging and their executive function and behavior using the Behavior Rating Inventory of Executive Function (BRIEF) assessment. Specifically, we measured brain cortical thickness and surface area, as well as behavioral regulation and Metacognition Indexes and other BRIEF subscales, and compared those parameters among the 3 diet groups. We also tested whether there are significant correlations between these MR imaging parameters and BRIEF test scores regardless of the infant diet group.

\section{MATERIALS AND METHODS}

\section{Subjects}

Healthy 7.5- to 8.5-year-old children were recruited for this brain power study (Infant Diet Effects on Brain Function and Language Processing; ClinicalTrials.gov Identifier: NCT00735423). All experimental procedures were approved by the institutional review board of the University of Arkansas for Medical Sciences, and all participants provided assent as well as parental consent. Inclusion criteria for the participants included the following: 90-101 months of age; parental report of full-term gestation at birth (38-42 weeks); parental report of birth weight between the 5 th and 95th percentile for age (2.5-4.2 kg); and parental report of predominant use of breast milk, milk-based formula, or soy-based formula during infancy. BF infants were exclusively or partially breastfed for at least 8 months (rounded to the closest number) before completely transitioning to formula or other diets; MF and SF infants were fed for same type of formula (cow's milk-based or soy-based) since the first weeks of life throughout the first year of life. Exclusion criteria for the participants included the following: maternal use of alcohol, tobacco, illicit drugs, or psychotropic medications during pregnancy; illnesses and chronic diseases that may affect children's growth or development; psychological/psychiatric diagnoses; neurologic impairment or injury; history or current use of anticonvulsant, stimulant, or mood-stabilizing medications; and history or current use of remedial special education services. In total, 71 children had valid structural MR imaging data and were included in this study. Among them, 68 children had completed BRIEF assessment by their parents. The demographic information of the study subjects is listed in Table 1.

\section{MR Imaging Data Acquisition}

All children had a brain MR imaging at the radiology department of the Arkansas Children's Hospital on a 1.5T Achieva scanner (Philips Healthcare) with a $60-\mathrm{cm}$ bore size, $33-\mathrm{mT} / \mathrm{m}$ gradient amplitude, and $100-\mathrm{mT} / \mathrm{m} / \mathrm{ms}$ maximum slew rate. The built-in body coil was used as a transmitter, and a standard 8-channel sensitivity encoding head coil was used as a receiver. Structural imaging data of the brain were acquired using a T1weighted 3D turbo field echo pulse sequence with the following parameters: $\mathrm{TR}=7.3 \mathrm{~ms}$; $\mathrm{TE}=3.4 \mathrm{~ms}$; flip angle $=8^{\circ}$; acquisition voxel size $=1 \times 1 \times 1 \mathrm{~mm}$; matrix size $=256 \times 232 \times$ 150; two averages; and 7 minutes of scan time. All images were reviewed on the scanner at the time of scanning, and scans with substantial motion artifacts were repeated.

\section{MR Imaging Processing}

All T1-weighted MR images were exported to a Macintosh computer with FreeSurfer software (http://surfer.nmr.mgh.harvard. edu) for cortical analysis. Standard preprocessing steps were applied, including motion correction, nonbrain tissue removal, and transformation to the Talairach space. Imaging segmentation (to white matter, gray matter, and CSF) was then performed, followed by intensity normalization, tessellation of cortical gray/ white matter boundaries, automated topology correction, and surface deformation. Cortical images were registered to a spheric atlas to match geometry across subjects and parcellated to different brain regions on the basis of anatomy. In addition, a full width half maximum Gaussian blurring kernel of $10 \mathrm{~mm}$ was applied to smooth the parameter maps. All processed or intermediate images were visually inspected to ensure quality. Cortical thickness and outer surface area were calculated, respectively, from distances between gray/white and gray/CSF boundaries and the area of all vertices along the surface. The cortical thickness 
Table 1: Demographic information of the 8-year-old children participating in the study

\begin{tabular}{|c|c|c|c|c|}
\hline & $\mathrm{BF}(n=22)$ & MF $(n=25)$ & $\operatorname{SF}(n=24)$ & $P$ Value \\
\hline $\operatorname{Sex}(M / F)$ & $10 / 12$ & $10 / 15$ & $11 / 13$ & .90 \\
\hline Weight (mean) (kg) & $27.8 \pm 6.3$ & $27.7 \pm 5.1$ & $27.2 \pm 4.8$ & .90 \\
\hline Height (mean) (cm) & $127.7 \pm 4.7$ & $128.5 \pm 4.1$ & $125.9 \pm 5.3$ & .16 \\
\hline Age at MR imaging (mean) (yr) & $7.98 \pm 0.29$ & $7.93 \pm 0.25$ & $7.87 \pm 0.25$ & .33 \\
\hline BRIEF completed (M/F) & $10 / 12$ & $10 / 13$ & $10 / 13$ & .99 \\
\hline Mother's education (no/partial or full/above college) & $0 / 9 / 5$ & $0 / 9 / 6$ & $1 / 6 / 7$ & .54 \\
\hline Father's education (no/partial or full/above college) & $2 / 10 / 2$ & $2 / 10 / 3$ & $3 / 9 / 0$ & .56 \\
\hline Mother's annual income $(<\$ 20,000 / \$ 20,000-\$ 59,999 />\$ 59,999)$ & $6 / 3 / 3 / 0$ & $12 / 3 / 7 / 2$ & $11 / 2 / 6 / 3$ & .52 \\
\hline Father's annual income $(<\$ 20,000 / \$ 20,000-\$ 59,999 />\$ 59,999)$ & $14 / 0 / 9 / 5$ & $15 / 0 / 8 / 7$ & $11 / 0 / 5 / 6$ & .63 \\
\hline
\end{tabular}

Table 2: Global imaging features for the 3 feeding groups

\begin{tabular}{llllcc}
\hline \multicolumn{1}{c}{ Features } & & & $\boldsymbol{F}$ & $\boldsymbol{P}$ \\
& BF (Mean) & MF (Mean) & SF (Mean) & Score & Value \\
\hline LH mean cortical thickness (mm) & $2.77 \pm 0.09$ & $2.76 \pm 0.09$ & $2.78 \pm 0.09$ & 0.30 & .74 \\
RH mean cortical thickness (mm) & $2.76 \pm 0.08$ & $2.75 \pm 0.10$ & $2.78 \pm 0.09$ & 0.64 & .53 \\
LH surface area $\left(\mathrm{cm}^{2}\right)$ & $905 \pm 83$ & $893 \pm 78$ & $933 \pm 104$ & 1.28 & .29 \\
RH surface area $\left(\mathrm{cm}^{2}\right)$ & $902 \pm 80$ & $897 \pm 83$ & $937 \pm 104$ & 1.43 & .25 \\
LH cortical volume $\left(\mathrm{cm}^{3}\right)$ & $291 \pm 25$ & $289 \pm 24$ & $300 \pm 31$ & 1.26 & .29 \\
RH cortical volume $\left(\mathrm{cm}^{3}\right)$ & $290 \pm 24$ & $289 \pm 26$ & $301 \pm 32$ & 1.50 & .23 \\
Total intracranial volume $\left(\mathrm{cm}^{3}\right)$ & $1490 \pm 127$ & $1502 \pm 123$ & $1529 \pm 149$ & 0.5 & .61 \\
\hline
\end{tabular}

Note:-LH indicates left hemisphere; $\mathrm{RH}$, right hemisphere.

and surface area parameter maps were then fed to a General Linear Model (GLM) in FreeSurfer for group comparisons.

\section{BRIEF Assessment}

The BRIEF is an 86-item questionnaire for parents that assesses executive function and behavior for their children and adolescents 5-18 years of age. ${ }^{15}$ The parents of children who completed MR imaging were requested to complete the BRIEF assessment. Eight clinical subscales were derived from the answers to the questionnaire, including Inhibit, Shift, Emotional Control, Initiate, Working Memory, Plan/Organize, Organization of Materials, and Monitor. These 8 subscales formed 2 indexes regarding the children's executive function and behavior: the Behavioral Regulation Index (BRI) and Metacognition Index, and 1 overall score: Global Executive Composite (GEC). In addition, we also obtained 2 validity scores: Negativity (reflects the extent to which the questionnaire was answered in an unusually negative manner) and Inconsistency (reflects the extent to which the respondent answers similar questions in an inconsistent manner). All BRIEF scores/ indexes/subscales were compared among the 3 diet groups, and their correlations with MR imaging-measured cortical thickness and surface area were also calculated.

\section{Statistical Analysis}

All comparisons of demographic parameters were conducted using the $\chi^{2}$ test for categoric data and the ANOVA with the distribution assumption test for quantitative data, and $P \leq .05$ was regarded as significant. Numeric data were presented as mean \pm SD. All comparisons of BRIEF scores among different diet groups were conducted using the Mann-Whitney $U$ test. All correlation tests between MR imaging parameters and BRIEF scores were conducted using the Spearman correlation test. For imaging parameters, all global features such as mean cortical thickness and surface area were compared among groups using a 1-way $F$ test. Group differences in regional imaging parameters (cortical thickness and surface area) were tested using GLM with the Different Offset, Different Slope (DODS) method in FreeSurfer. Specifically, to test the diet group differences, we used GLMs with DODS to fit in each vertex for cortical thickness or cortical surface area, which was a dependent variable. The feeding type $(\mathrm{BF}$, $\mathrm{MF}$, or SF) was used as an independent variable, and sex and age were included as covariates because of reported sex differences ${ }^{16}$ and continued brain cortical development ${ }^{17}$ at this age. Total brain volume was included as a covariate in the surface area analyses but not in the cortical thickness analyses because a previous report showed that normalizing by intracranial volume did not improve the discriminant and predictive performance of cortical thickness measures. ${ }^{18}$ All analyses were applied to each hemisphere separately. The $P \leq .0001$ threshold was used for cluster forming for the vertex-wise analyses. Clusters were obtained after removing the effects of all covariates. To identify clusters with significant differences after appropriate multiple comparison correction, we applied the precached cluster-wise Monte Carlo simulation with 10,000 iterations to every cluster. A corrected cluster-wise $P \leq .05$ was regarded as significant. In addition, stratified analyses were also applied to the boys' and girls' subgroups, respectively.

\section{RESULTS}

\section{MR Imaging Findings}

There were no significant differences in the demographic characteristics across the 3 feeding groups (Table 1). No significant group effects among BF/MF/SF groups were observed for global imaging features of the brain such as mean cortical thickness, total surface area, total cortical gray matter volume, and intracranial volume. Details are listed in Table 2.

In total, 8 clusters showed significant infant diet-related differences ( $P \leq .05$, after multiple comparison correction) in the combined or sex-specific regional analyses of cortical thickness and surface area, as summarized in Table 3, and location as illustrated in On-line Fig 1. Specifically, higher cortical thickness $(P \leq .05$, corrected) was found in a cluster in the right cuneus (cluster 1) when comparing all MF-versus-all BF children. It appears that the differences in this cluster were primarily driven by differences in 
Table 3: Clusters that showed significant differences $(P \leq .05$, corrected) in group comparisons of cortical thickness or surface $\operatorname{area}^{a}$

\begin{tabular}{|c|c|c|c|c|c|c|c|c|}
\hline \multirow[b]{2}{*}{ Cluster No. } & \multirow[b]{2}{*}{ Size $\left(\mathrm{mm}^{2}\right)$} & \multirow{2}{*}{$\begin{array}{c}\text { No. of } \\
\text { Vertices }\end{array}$} & \multicolumn{3}{|c|}{ Peak Coordinate } & \multirow{2}{*}{$\begin{array}{c}\text { Peak Vertex } \\
t \text { Value }\end{array}$} & \multirow{2}{*}{$\begin{array}{c}\text { Cluster-Wise } \\
P \text { Value }\end{array}$} & \multirow[b]{2}{*}{ Relationships } \\
\hline & & & $x$ & $y$ & z & & & \\
\hline 1 & 106 & 134 & 9.9 & -87.1 & 17.7 & 5.05 & .007 & All: MF>BF \\
\hline 2 & 132 & 165 & 10.7 & -87.4 & 18.5 & 5.38 & .003 & Boys: $M F>B F$ \\
\hline 3 & 128 & 180 & -11.4 & -74 & 12.7 & 5.34 & .004 & Boys: $M F>B F$ \\
\hline 4 & 151 & 189 & 11.2 & -87.8 & 18.5 & 4.76 & .001 & Boys: $M F>S F$ \\
\hline 5 & 78 & 100 & 3.6 & -74.2 & 14.3 & 4.88 & .02 & Boys: $M F>S F$ \\
\hline 6 & 87 & 82 & -7.8 & -87.9 & -10.8 & 4.65 & .01 & Boys: $M F>S F$ \\
\hline 7 & 53 & 117 & 15.2 & -16 & 64.5 & 4.38 & .046 & Girls: SF $>M F$ \\
\hline 8 & 138 & 220 & -32.9 & 8.5 & 55.3 & 4.77 & .01 & Boys: $M F>S^{a}$ \\
\hline
\end{tabular}

${ }^{a}$ For surface area comparison; everything else: for cortical thickness comparison.

the male subgroups because a similar cluster (cluster 2) showed higher cortical thickness $(P \leq .05$, corrected) in MF-versus-BF boys, while there were no significant differences between MF and BF girls. In addition, a small cluster in the left pericalcarine cortex (cluster 3) also showed higher cortical thickness $(P \leq .05$, corrected) in MF-versus-BF boys. When comparing all MF-versus-all SF children, there were no significant differences between groups in any regions. However, sex-specific analyses showed that 2 clusters (clusters 4 and 5) in the right cuneus and 1 cluster in the left lingual gyrus (cluster 6) showed higher cortical thickness $(P \leq .05$, corrected) in MF-versus-SF boys, while 1 cluster in the right precentral gyrus (cluster 7) showed lower cortical thickness $(P=.046)$ in MF-versus-SF girls. There were no significant differences in cortical thickness for BF-versus-SF comparisons (combined or sexspecific). Finally, higher surface area $(P \leq .05$, corrected) was also observed in a cluster in the left middle frontal gyrus (cluster 8) when comparing MF-versus-SF boys, while no other group differences in surface area were found in the combined or sex-specific analyses.

\section{BRIEF Assessment Findings}

None of the children for any diet groups showed clinically significant symptomatology on the BRIEF assessment (GEC score or Metacognition Index/BRI index of $>65)$. The mean Negativity $(0.1 \pm 0.3$ for $\mathrm{BF}, 0.1 \pm 0.5$ for $\mathrm{MF}, 0 \pm 0$ for $\mathrm{SF})$ and Inconsistency scores $(2.3 \pm 1.6$ for BF, $2.8 \pm 1.4$ for MF, $2.3 \pm 1.9$ for SF) were low and below the validity threshold, and there were no differences among the 3 diet groups $(P>.05$ for any comparison). Other BRIEF assessment scores are presented in On-line Fig 2. Specifically, the overall mean GEC scores were not different in BF versus MF or BF versus SF but were significantly higher in MF versus SF (46.8 \pm 6.7 versus $42.4 \pm 6.4$, $P=.049$ ). The differences were driven by differences in boys (sex-specific analyses showed $45.3 \pm 3.6$ versus $38.6 \pm 4.9$, $P=.006$ for boys, and $48.0 \pm 8.4$ versus $45.4 \pm 6.0, P=.55$ for girls). Likewise, the mean Metacognition Indexes were not different in BF versus MF or BF versus SF but were significantly higher in MF versus SF (47.6 \pm 7.0 versus $42.7 \pm 7.6, P=.016$ ), driven by differences in boys $(45.5 \pm 5.0$ versus $38.4 \pm 5.6$, $P=.008)$ but not girls $(49.2 \pm 8.1$ versus $46.0 \pm 7.4, P=.21)$. The mean BRI indexes were not different for the BF/MF, BF/SF, or $\mathrm{MF} / \mathrm{SF}$ comparisons. However, there was a trend of lower BRI scores in SF boys (40.2 \pm 5.9$)$ compared with MF boys
(45.3 $\pm 4.5, P=.06)$ or BF boys $(47.4 \pm 9.0, P=.06)$. For the 8 specific BRIEF clinical subscales, SF boys had lower scores compared with MF boys in Shift $(P=.047)$, Initiate $(P=.004)$, Plan/ Organize $(P=.02)$, and Monitor $(P=.02)$, which contributed to lower scores for all SF versus all MF in Initiate $(P=.01)$, Plan/ Organize $(P=.03)$, and Monitor scores $(P=.007)$, despite no significant differences between SF and MF girls in any of these 8 measures. None of the BF/SF or BF/MF comparisons showed significant differences in any of these 8 measures. Overall, while all children had BRIEF scores within the normal range, SF boys showed less parent-reported executive functioning or behavior issues.

\section{Correlations between MR Imaging Measurements and BRIEF Scores}

For the clusters that showed significant differences in regional cortical thickness or surface area among different diet groups (see the MR Imaging Findings section above), the correlations between these morphometric features with each of the BRIEF scores/indexes/subscales were also tested. For boys, the overall GEC scores (On-line Fig 3) significantly correlated with cluster cortical thickness in cluster $1(r=0.51, P=.004)$, cluster 2 $(r=0.54, P=.002)$, cluster $4(r=0.56, P=.001)$, and cluster 6 $(r=0.41, P=.02)$. There were no significant correlations between GEC scores and cortical thickness or surface area in other clusters (On-line Table). For girls, there were no significant correlations between GEC scores and any cluster features (cortical thickness or surface area). Similarly, for the BRI and Metacognition indexes and the 8 BRIEF subscales, significant correlations with cortical thickness in clusters 1, 2, 4, and 6 were observed in a number of tests for boys, while no significant correlations were observed for girls (except for correlation between working memory score and cortical thickness in cluster 6, $r=0.38, P=.02)$ (On-line Table).

\section{DISCUSSION}

The first year of life is a critical period for normal brain development. Total brain volume doubles in the first year, which includes a $149 \%$ increase in gray matter volume and an $11 \%$ increase in white matter volume. ${ }^{19}$ Influences on the brain development during this time, such as those associated with infant diet, may have profound and prolonged effects on long-term brain development and neurodevelopmental outcomes. In this study, we evaluated brain cortical 
thickness and surface area measurements by neuroimaging in 3 groups of 8-year-old children who were predominately BF, MF, or SF during infancy. While there were no group differences on the whole-brain average of these imaging features, we observed significant differences in cortical thickness and surface area associated with infant diets in several clusters in the brain cortex. These findings were mostly attributed to differences in boys but not girls. Likewise, we also observed group differences in assessments of executive function and behavior in these children, with significant findings in boys but not in girls. In particular, SF boys showed lower cortical thickness and smaller cortical surface area in several regions of the brain. Meanwhile, SF boys showed lower parent-reported BRIEF scores compared with MF boys, while MF and BF groups had comparable BRIEF scores for both boys and girls. There were also significant correlations between overall BRIEF scores and mean cortical thickness in several of the cortical clusters that showed significant group differences associated with infant diets, indicating potential structural-functional relationships for these brain regions. In summary, our findings suggested that infant diets may have long-term effects on children's brain structural development and functional outcomes, particularly for boys who were predominately fed milk or soy protein-based formulas. Nevertheless, despite statistically significant regional cortical differences in SF and MF boys in the MR imaging data, the BRIEF data for all children were within the normal range, and formula feeding per se during infancy did not result in clinically significant executive function and behavior abnormalities at 8 years of age.

Brain developmental differences associated with different infant diets such as breastfeeding versus formula feeding have been reported in recent years. ${ }^{7-10,20}$ The reported effects apparently were also more prominent in boys versus girls because some of these studies only observed diet-associated brain differences in boys but not in girls. For example, fractional anisotropy, a reflection of white matter microstructural integrity (such as myelination), was significantly higher in several white matter regions in breastfed-versusformula fed boys, while it was similar in girls. ${ }^{9}$ In addition, the percentage of expressed maternal breastmilk in the infant diet positively correlated with total white matter volume in boys but not in girls, though the cohort studied were all born preterm. ${ }^{6}$ Benefits to neurodevelopmental outcomes associated with breastfeeding have also been widely reported, and a large-scale randomized controlled trial (randomization to a breastfeeding promotion intervention program targeted at increasing breastfeeding exclusivity and the duration among those already having decided to initiate breastfeeding) did observe a slightly higher treatment effect in verbal intelligence quotient for boys versus girls (8.0- versus 7.0-point increase for the intervention group). ${ }^{5}$

Beyond infant diets, it also appears that in general, the developing brain in boys is more vulnerable to adverse environmental influences and early life experience; ${ }^{21}$ this vulnerability may point to a sex-specific difference in brain malleability in response to early life events. One main finding of this study was significant differences in brain cortical development and executive function and behavior between SF and MF boys but not girls. The exact underlying mechanisms for this observation remain to be uncovered. One speculation is that the high concentration of phytoestrogens/isoflavones contained in soy milk may have played a role. SF infants have circulating phytoestrogen concentrations several magnitudes higher than endogenous estrogens. Although the potency of phytoestrogens is significantly lower than endogenous estrogens, the presence of circulating soy phytoestrogens may account for the effects observed herein. This possibility would be in agreement with reports in lab animals suggesting that phytoestrogens may interfere with the organizational role of estrogen in the developing human brain. ${ }^{22}$ While the effects of endocrine disruption on sexually dimorphic brain regions in rodents have been extensively studied, how estrogenic compounds would impact sex-specific changes in human brain development is not as clear. Nevertheless, it is known that male and female brain regions respond differently to estrogens. ${ }^{23}$ Therefore, it is possible that the assumed estrogenic effects of soy formula feeding on the infant brain and neurodevelopment were sex-specific. In fact, clinical studies of the effects of soy isoflavones on cognitive function in adults have shown improvement in cognitive functions for women, but results for men have been inconsistent, ${ }^{24-27}$ also suggesting that the putative soy isoflavone effects may be sex-specific.

The brain regions showed that infant diet-associated effects on cortical thickness or surface area in these 8-year-old children mostly involved the cuneus and the pericalcarine cortex. These are areas usually regarded as the primary visual cortex, with a main function of basic visual processing. Other brain regions that showed infant diet-related effects included the following: the lingual gyrus, which is also linked to visual processing as well as encoding visual memories; the precentral gyrus, commonly known as the primary motor cortex and controls voluntary movement; and the middle frontal gyrus, which is implicated in a number of brain functions, such as language and cognition. Previous studies reporting associations between infant diets (particularly breastfeeding) and children's brain cortical development (such as cortical thickness or regional gray matter volume) have observed significant effects primarily in the parietal and temporal lobes. ${ }^{7,10}$ Our study revealed additional brain regions in the frontal and occipital lobes that were also impacted by infant diets, indicating possibly widespread and profound effects of early nutrition on children's brain development.

Brain regions with diet effects on cortical measurements observed in this study were different from those in a previous study of 8 -year-old children focused on regional gray matter volume. ${ }^{10}$ It is possible that methodologic differences in these studies contributed to the apparent discrepancy. For example, regional gray matter volume measurements are often normalized to total brain volume, while cortical thickness measurements may be more sensitive without normalization. ${ }^{18}$ The sensitivity of cortical thickness to developmental changes may also be impacted in some brain regions by the competing effects of initial development during infancy and normal thinning, which starts in early childhood. The regions observed in this study were primarily involved in visual, motor, and language/ cognitive functioning, respectively. While breastfeeding versus formula feeding did not appear to significantly impact children's visual outcome ${ }^{28}$ exclusive breastfeeding or longer breastfeeding duration was favorably associated with physical fitness, ${ }^{29}$ language development, ${ }^{30}$ and cognition/intelligence in children. ${ }^{31}$ In addition, we observed significant correlations between cortical thickness in visual processing areas in the occipital lobe (cuneus and lingual gyrus) 
and executive function and behavior evaluated by the BRIEF assessment. While the exact structural/functional relationship of this finding is unclear, the ability to search and process visual information is a prerequisite for many executive function. Furthermore, animal studies $^{32}$ and human studies ${ }^{33}$ have shown the effects of soy isoflavones on visual memory, which may be a reflection of structural influences, particularly in the visual pathways. Overall, the areas that showed significant differences in cortical thickness or surface area in this study were quite small, and the biologic meaning based on these results remains unclear and will need further investigation.

The main limitation of this study is that the study subjects were recruited at 8 years of age, and the infant diet pattern was self-reported by parents. While parents reported the dominant feeding type (BF, MF, or SF), some of the other important nutritional information is missing, such as the precise nutrient component of the diets, exclusivity of feeding type, and amount of intake per feeding. In addition, while we controlled for age and sex in the data analysis, additional potential confounding factors were not controlled, such as nutrition and lifestyle after infancy and other postnatal factors (limited by the retrospective study design and lack of methods to quantify), possibly impacting children's brain development and neurodevelopmental outcomes as well. Also, family environment and socioeconomic status, which is likely another potential confounder to be considered, was only compared among groups (no significant differences were found) but not included as a covariate in the data analysis due to incomplete data. In this study, we focused on cortical measurements, while other important brain features such as microstructural development and connectivity were not studied. The sample size, if breaking down to each diet group and each sex, was also relatively small; and the parent-reported BRIEF assessment was an indirect measure of executive function. Follow-up MR imaging and neurodevelopmental evaluations on a larger and prospective cohort of children with different infant diets are underway, with key potential covariates carefully measured. This follow-up study will be helpful to confirm the findings in the current report.

\section{CONCLUSIONS}

The composition of the typical choices available for an infant's diet (ie, BF, MF, SF) may have profound and long-term effects on children's brain development, which can be reflected by brain imaging and executive function assessment at 8 years of age. The effects appear to be sex-specific, with boys more likely to show diet-associated effects than girls. The findings support the idea that soy-based formula may alter later life brain anatomy and function but that any changes are modest and do not lead to clinically relevant deficits or abnormal outcomes.

\section{ACKNOWLEDGMENT}

We thank the staff of the Arkansas Children's Nutrition Center Clinical Research Core for their assistance with this study.

Disclosures: Ting Li-UNRELATED: Employment: University of Arkansas at Little Rock. Thomas M. Badger-RELATED: Grant: USDA-ARS, Comments: research grant funded. Xiangyang Lou-RELATED: Grant: National Science Foundation.* Xiawei Ou-RELATED: Grant: US Department of Agriculture, Comments: The work presented in this article is funded by a US Department of Agriculture grant*; UNRELATED: Grants/Grants Pending: US Department of Agriculture, Comments: The authors are supported by a number of National Institutes of Health/US Department of Agriculture grants. * *Money paid to the institution.

\section{REFERENCES}

1. Johnston M, Landers S, Noble L, et al. Breastfeeding and the use of human milk. Pediatrics 2012;129:E827-41 CrossRef Medline

2. Breastfeeding Report Card, United States. 2018. https://www.cdc.gov/ breastfeeding/data/reportcard.htm.

3. Bhatia J, Greer F, Comm N; American Academy of Pediatrics Committee on Nutrition. Use of soy protein-based formulas in infant feeding. Pediatrics 2008;122:1062-68 CrossRef Medline

4. Lucas A, Morley R, Cole TJ, et al. Breast-milk and subsequent intelligence quotient in children born preterm. Lancet 1992;339:261-64 CrossRef Medline

5. Kramer MS, Aboud F, Mironova E, et al; Promotion of Breastfeeding Intervention Trial (PROBIT) Study Group. Breastfeeding and child cognitive development: new evidence from a large randomized trial. Arch Gen Psychiatry 2008;65:578-84 CrossRef Medline

6. Isaacs EB, Fischl BR, Quinn BT, et al. Impact of breast milk on intelligence quotient, brain size, and white matter development. Pediatr Res 2010;67:357-62 CrossRef Medline

7. Kafouri S, Kramer M, Leonard G, et al. Breastfeeding and brain structure in adolescence. Int J Epidemiol 2013;42:150-59 CrossRef Medline

8. Deoni SCL, Dean DC, Piryatinsky I, et al. Breastfeeding and early white matter development: a cross-sectional study. Neuroimage 2013;82:7786 CrossRef Medline

9. Ou X, Andres A, Cleves MA, et al. Sex specific association between infant diet and white matter integrity in eight-year-old children. Pediatr Res 2014;76:535-43 CrossRef Medline

10. Ou X, Andres A, Pivik RT, et al. Voxel-based morphometry and fMRI revealed differences in brain gray matter in breastfed and milk formula-fed children. AJNR Am J Neuroradiol 2016;37:713-19 CrossRef Medline

11. Crider A, Pillai A. Estrogen signaling as a therapeutic target in neurodevelopmental disorders. J Pharmacol Exp Ther 2017;360:48-58 CrossRef Medline

12. Lee YB, Lee HJ, Sohn HS. Soy isoflavones and cognitive function. $J$ Nutr Biochem 2005;16:641-49 CrossRef Medline

13. Jing HK, Gilchrist JM, Badger TM, et al. A longitudinal study of differences in electroencephalographic activity among breastfed, milk formula-fed, and soy formula-fed infants during the first year of life. Early Hum Dev 2010;86:119-25 CrossRef Medline

14. Pivik RT, Andres A, Badger TM. Effects of diet on early stage cortical perception and discrimination of syllables differing in voice-onset time: a longitudinal ERP study in 3- and 6-month-old infants. Brain Lang 2012;120:27-41 CrossRef Medline

15. Gioia GA, Isquith PK, Guy SC, et al, eds. Behavior Rating Inventory of Executive Function (BRIEF). Psychological Assessment Resources; 2000

16. De Bellis MD, Keshavan MS, Beers SR, et al. Sex differences in brain maturation during childhood and adolescence. Cereb Cortex 2001;11:552-57 CrossRef Medline

17. Giedd JN, Rapoport JL. Structural MRI of pediatric brain development: what have we learned and where are we going? Neuron 2010;67:728-34 CrossRef Medline

18. Westman E, Aguilar C, Muehlboeck JS, et al. Regional magnetic resonance imaging measures for multivariate analysis in Alzheimer's disease and mild cognitive impairment. Brain Topogr 2013;26:9-23 CrossRef Medline

19. Knickmeyer RC, Gouttard S, Kang CY, et al. A structural MRI study of human brain development from birth to 2 years. J Neurosci 2008;28:12176-82 CrossRef Medline

20. Isaacs EB. Neuroimaging, a new tool for investigating the effects of early diet on cognitive and brain development. Front Hum Neurosci 2013;7:445 CrossRef Medline 
21. Schore AN. All our sons: the developmental neurobiology and neuroendocrinology of boys at risk. Infant Ment Health J 2017;38:15-52 CrossRef Medline

22. Patisaul HB, Jefferson W. The pros and cons of phytoestrogens. Front Neuroendocrinol 2010;31:400-19 CrossRef Medline

23. Gillies GE, McArthur S. Estrogen actions in the brain and the basis for differential action in men and women: a case for sex-specific medicines. Pharmacol Rev 2010;62:155-98 CrossRef Medline

24. Kritz-Silverstein D, Von Muhlen D, Barrett-Connor E, et al. Isoflavones and cognitive function in older women: the SOy and Postmenopausal Health In Aging (SOPHIA) Study. Menopause 2003;10:196-202 Medline

25. Duffy $\mathrm{R}$, Wiseman H, File SE. Improved cognitive function in postmenopausal women after 12 weeks of consumption of a soya extract containing isoflavones. Pharmacol Biochem Behav 2003;75: 721-29 CrossRef Medline

26. White LR, Petrovitch $\mathrm{H}$, Ross GW, et al. Brain aging and midlife tofu consumption. J Am Coll Nutr 2000;19:242-55 CrossRef Medline

27. File SE, Jarrett N, Fluck E, et al. Eating soya improves human memory. Psychopharmacology (Berl) 2001;157:430-36 CrossRef Medline
28. Rudnicka AR, Owen CG, Richards M, et al. Effect of breastfeeding and sociodemographic factors on visual outcome in childhood and adolescence. Am J Clin Nutr 2008;87:1392-99 CrossRef Medline

29. Tambalis KD, Mourtakos S, Panagiotakos DB, et al. Exclusive breastfeeding is favorably associated with physical fitness in children. Breastfeed Med 2019;14:390-97 CrossRef Medline

30. Belfort MB, Rifas-Shiman SL, Kleinman KP, et al. Infant feeding and childhood cognition at ages 3 and 7 years: effects of breastfeeding duration and exclusivity. JAMA Pediatr 2013;167:836-44 CrossRef Medline

31. Horta BL, de Mola CL, Victora CG. Breastfeeding and intelligence: a systematic review and meta-analysis. Acta Paediatr 2015;104:1419 CrossRef Medline

32. Lund TD, West TW, Tian LY, et al. Visual spatial memory is enhanced in female rats (but inhibited in males) by dietary soy phytoestrogens. BMC Neurosci 2001;2:20 CrossRef Medline

33. Gleason CE, Carlsson CM, Barnet $\mathrm{JH}$, et al. A preliminary study of the safety, feasibility and cognitive efficacy of soy isoflavone supplements in older men and women. Age Ageing 2008;38:86-93 CrossRef Medline 